\title{
AD HOC BASED VEHCULAR NETWORKING AND COMPUTATION
}

\author{
Zeeshan Osmani $^{1}$, Shoaib Khan ${ }^{2}$, Saif $\mathrm{Khan}^{3}$, Awij Shaikh \\ 1, 2, 3,4 Department of Electronics \& Communication Engineering, M.H, Saboo Siddik College of Engineering \\ Maharashtra, India
}

\begin{abstract}
Vehicular Ad Hoc network which is a highly wireless network has went through lot of research in the past few years. The VANETs services are being used in many services today but because of many difficulties faced due to connectivity issues faced and low scalability the VANETs have yet not become popular. The existing VANET routing protocols need a topology that can serve as a framework which will help in its integration. For validating our computation we used urban city model, and generated vehicular mobility traces from the mobility model. The traces were added to the network simulator to study the routing behaviour. To realise the safety feature of VANETs two scenarios are added, the regular safety data streaming and the accidental scenario streaming. The simulation results are demonstrated for the VANETs networking and computation.
\end{abstract}

Keywords: Vehicular Ad Hoc Networks (VANETs), Integration and Frame-Work.

\section{INTRODUCTION}

Vehicular Ad Hoc Networks are being planned to support various services such as route planning, traffic alert system, etc. A lot of consideration could be given to VANETs system and already it has gained attention research community. In VANET system the nodes are the vehicles, the important feature of VANET is these high mobility nodes will not only communicate with each other but also the road side unit also.

\section{COMMUNICATION}

Today the main challenges faced by VANET is the communication issues and the Quality of service between Vehicle to vehicle and vehicle to infrastructure.

\subsection{Vehicle to Vehicle Communication}

The nodes which are vehicles in the VANET system communicate with each other using various techniques including multi-hop technique in which the packets are hopped multiple times between the source and the destination. First the source point and destination point are selected, the vehicles in the path of the destination passes the packets to the forward vehicles unless the destination is reached. In this scenario two messages are send, timely safety update message and the other is event driven message. In the Safety message is for the user to follow the safety procedures at the time of driving. The other event driven message is to inform about any accident events or other issues to avoid the traffic near the area affected.

\section{$* * *$}

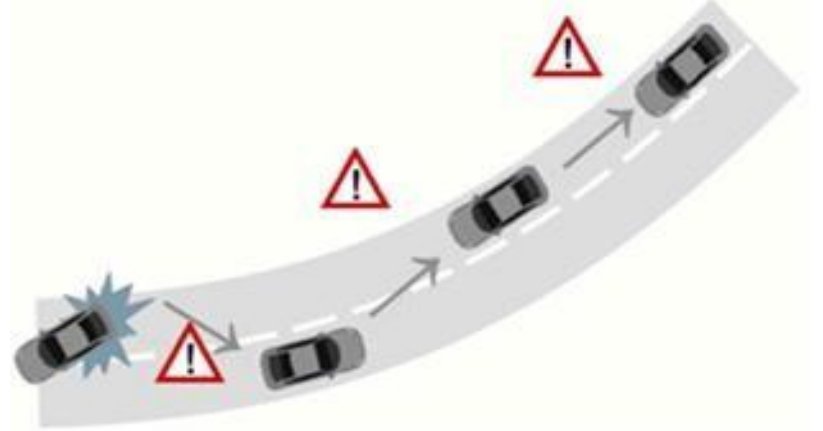

Figure 1. Vehicle to Vehicle Communication

\subsection{Vehicle to RSU Communication}

In this case the vehicles communicate with the Road Side Unit within the range of RSU. When the nodes are travelling along the road they exchange packets.

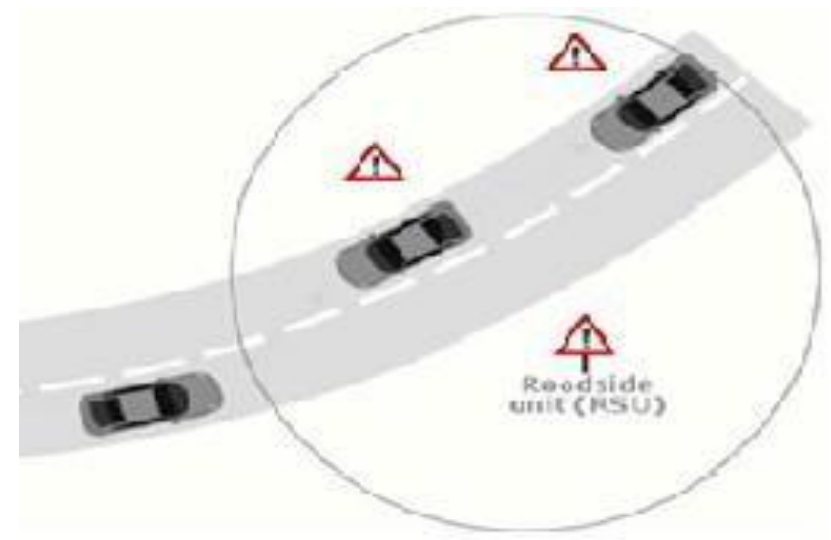

Figure 2.Vehile to RSU communication

\section{VANET model for Intersection of Roads}

It plots the intersection of road on both the stretches. It is assumed by the model that nodes are originated at random points with independent velocities at each point. 
MATLAB code was used to simulate this model. Developing the code was done to exploit the fact that the distance travelled by each node during the encounter will be much higher as compared to the computational speed of each location and the difference was of the order $0 . \mathrm{ms}$ to 10s. The nature of MATLAB code did not affect the accuracy of the model.

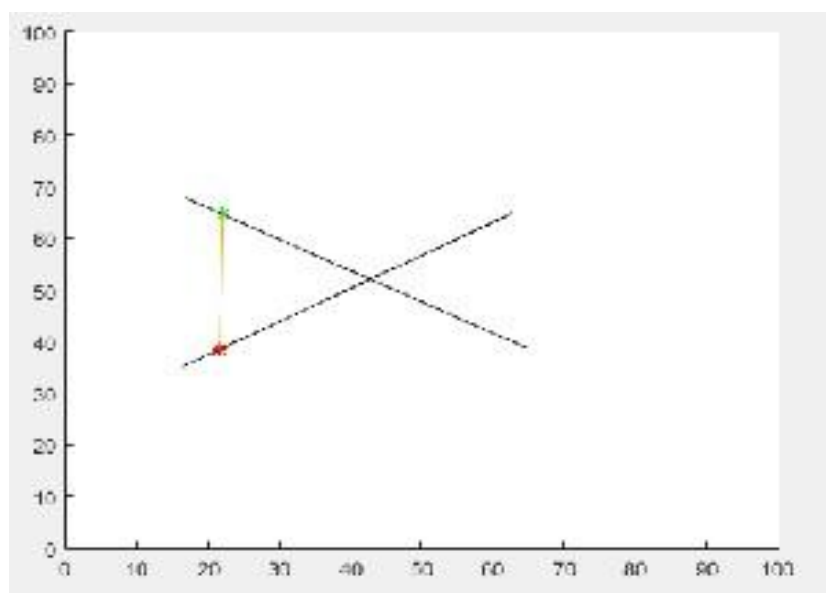

Figure 3. Intersection of Roads

\section{VANET MODEL FOR TWO SEPARATE}

\section{ROADS}

In this model of VANET two separate roads are there and separate nodes are travelling through the stretches. In the MATLAB code the two nodes are travelling through their respective roads with the same velocity. They originated at the same time and reach their destination at the same time.

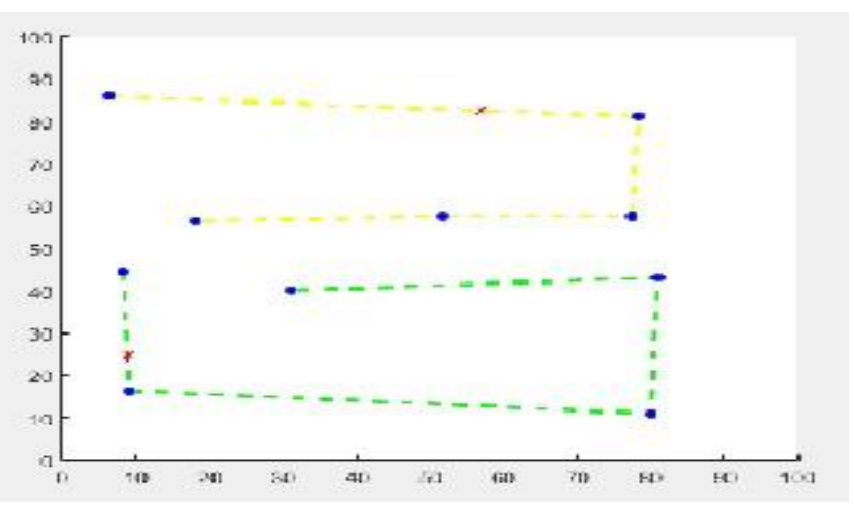

Figure 4. Two Separate Roads.

\section{VANET model for RSU}

In this model VANET nodes are passing through RSUs. It is similar to handoff of mobile station from one base station to another. The exchange of packet is carried out between RSU unit and the nodes and there is central Switching centre between the two roadside infrastructure Unit. The mechanism that involve the continuous connection maintenance over large distance in VANET system can be studied by this model.

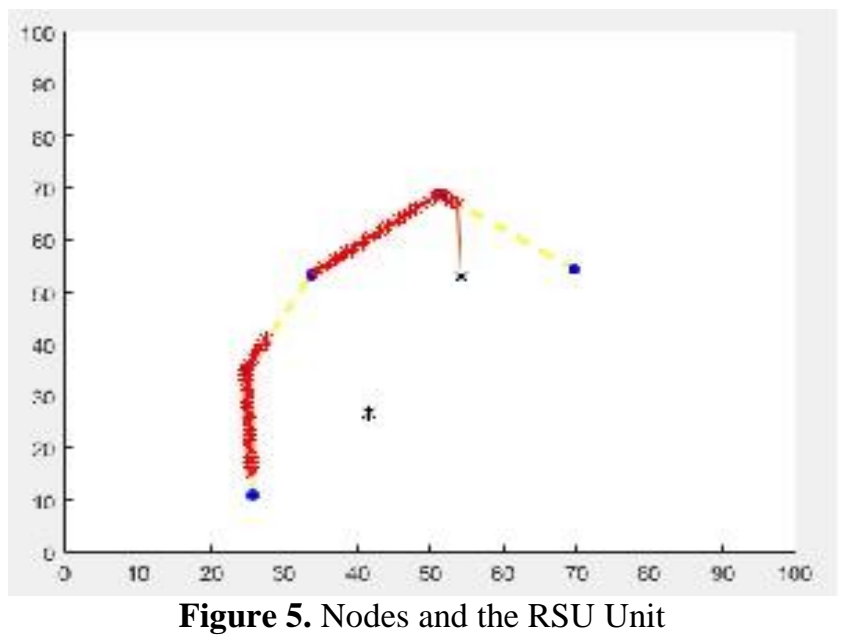

\section{VANET MODEL FORURBAN CITY}

This creates an entire network of roads instead of custom roads on a macro scale. It is modelled and used by nodes to populate and simulate. At the time of generation of nodes its direction is randomly chosen. The model formed by the nodes is in rectangular format which represent a block of house. This structure of rectangular roadway which is occupied by nodes are generated using the in-built MATLAB function.

This model created by using MATLAB code can be used for studying various aspects of the VANET systems.

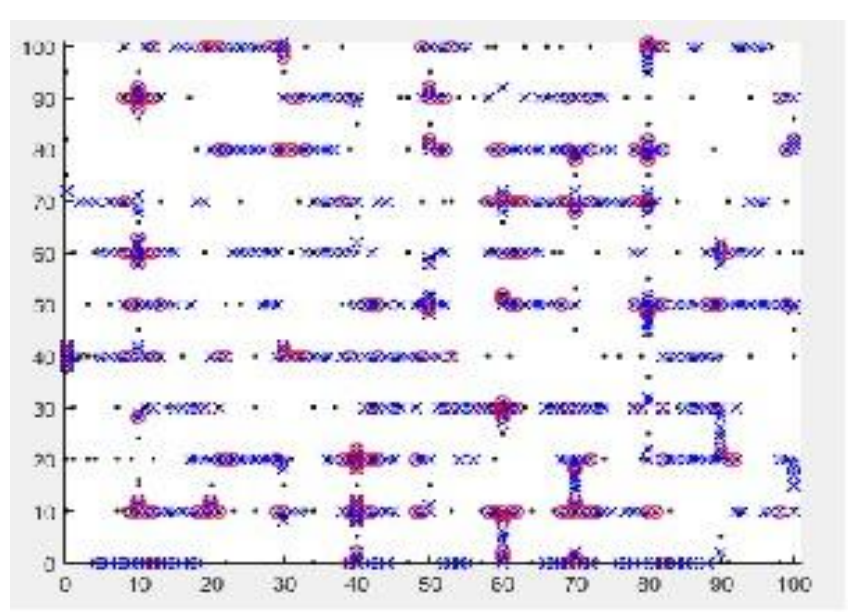

Figure 6. Urban City Simulation

\section{PROBLEM AND DRAWBACK}

The Vehicular Ad Hoc network is still not emerged as a vast technology, it is in its research stage of development. The connectivity problem, low scalability, large overhead are the issues to be resolved. The traffic laws, geometrical and geographical constraints bound the speed and direction of the nodes. The system associated with vehicular design, VANETs must be thoroughly tested before they are put in real world because the consequences of failing system are very high in VANETs. A proposed framework for VANET can make the technology going, also the mobility and generation topologies can serve as a tool for the development of technology. 


\section{RESULT}

There are large no of simulator tools available for study of VANET models like Fleet-Net, NHTSA and MATLAB. Of all simulator tools MATLAB is the most widespread, widely available and it is continuously updated. Hence we have simulated our project on MATLAB and it provides us unique advantages over other systems

\section{CONCLUSION}

The scenarios of various VANET model were simulated and computed. These developed simulative models can be used as a basis for further researches and tests. We expect that the researchers will get extra ordinary result for their efforts.

\section{REFERENCES}

[1]. Maen M. Artimy, William Robertson, and William J. Phillips, "Vehicular Ad Hoc Networks: An Emerging Technology Toward Safe and Efficient Transportation"

[2]. Grzybek, Agata; Seredynski, Marcin ;Danoy, Grégoire ; Bouvry, Pascal, "Aspects and trends in realistic VANET simulations", World of Wireless, Mobile and MultimediaNetworks (WoWMoM), 2012 IEEE International Symposium 2012

[3]. Sandeep Tayal, Malay Ranjan Tripathy, "VANET Challenges in selection of Vehicular Mobility Model", 2012 Second International Conference on Advanced Computing \& Communication Technologies

[4]. Marco Fiore, "Vehicular Mobility Simulation for VANETs", Simulation Symposium, 2007. ANSS '07. 40th Annual

[5]. Rahul Mangharam et al, "GrooveSim: a topography accurate simulator for geographic routing in vehicular networks", VANET '05 Proceedings of the 2nd ACM international workshop on Vehicular ad hoc networks 